\title{
nature
}

5 July 2001 Volume 412 Issue no 6842

\section{Blessings of mixed neutrinos}

The confirmation that neutrinos have mass and can switch identity is a triumph of careful experiment that opens doors for theoretical physicists. It is not a crisis for existing models, but a route to deeper ones.

$\mathrm{T}$ hanks to 1,000 tonnes of exquisitely pure deuterium oxide, two kilometres or so beneath Ontario, we now have hard evidence that the three 'flavours' of neutrino $\left(\nu_{\mathrm{e}}, \nu_{\mu}\right.$ and $\left.\nu_{\tau}\right)$ change identity - or oscillate - as they travel from the Sun to the Earth. As John Bahcall describes on page 29, new measurements from the Sudbury Neutrino Observatory, combined with those from the Super-Kamiokande detector in Japan reported in 1998, beautifully resolve a question that has stood unanswered for three decades: whether a deficit in the detected number of solar neutrinos was due to a crisis in the otherwise successful modelling of the Sun and stellar evolution, or to some identity crisis of neutrinos. The solar models are triumphant. Another debate is also resolved: physics tells us that this also means that neutrinos have mass — but, at less than about $10^{-7}$ of the mass of an electron, not enough to account for all of the mysterious dark matter that pervades the Universe.

But is the result a crisis for neutrino physics, and for high-energy physics more generally? High-energy theorists would have welcomed a crisis any time in the past decade. During that time, the theory that amalgamates models of the strong and weak nuclear forces and those of electromagnetic forces has successfully withstood all experimental challenges. This 'standard model' is one of the great intellectual triumphs of last century: it takes some simple fundamental principles of physical symmetries in space-time, some not-sosimple hypothetical 'internal symmetries' between particle properties, and also the basic postulates of quantum mechanics such as Schrödinger's equation, which links a particle's behaviour, the energy potentials within which it moves and the uncertainty principle. From those, it arrives at a world in which the known fundamental particles arise as excitations of fields, including one, the Higgs field, which gives rise to yet-unseen Higgs particles that interact with known particles to imbue them with mass.

\section{Beyond the standard model}

Given its success, whatever comes next, the standard model will be no more fundamentally right or wrong than is Newton's gravitational law, which has been supplanted by general relativity but still provides the basis of successful planetary missions. As with Newton's law, everyone is sure that the standard model is inadequate for explaining more extreme conditions. But, unlike Einstein, nobody has yet convinced everybody that they know just where, theoretically, to go next, although there are many ideas deposited weekly, if not daily, on the Los Alamos preprint server. The critical clues seem more likely to arise not from an unexpectedly falsified prediction of the standard model, but from phenomena discovered in regimes where the standard model is expected to be inadequate.

The fact that neutrinos have mass, therefore, is in no way a crisis for the standard model. Theorists differ about exactly how to represent such masses. Some explanations amount to a small extension of the model; others are more fundamentally different, and are expressed in more broadly predictive frameworks, with a higher degree of mathematical symmetry. These incorporate the standard model at the cost, or benefit, of predicting new particles or new interchangeability between particles. However, everyone agrees that each of the neutrino flavours observed must be represented as a superposition - a quantum mixture - of underlying basic states, which themselves are the mass-possessing outcomes of interactions with some sort of Higgs field. The standard model is already comfortable with the fact that such mixing can occur. What now needs to be measured or inferred by the Sudbury Neutrino Observatory, and others too, is the nature of the mixing, and the patterns of oscillation not only in empty space, but also, for example, in the interior of the Sun and in other astrophysical environments.

\section{Tow ards grand unific ation}

Several leading theorists, including Frank Wilczek (see Nature 391, 123-124; 1998) and John Ellis (see http://xxx.lanl.gov/abs/ hep-ph/0008334), see neutrino masses as one of several pointers to a Grand Unified Theory (GUT) - a seamless unification of the three forces. The nature of the mixing might help to identify just what sort of GUT explicitly describes not only the existence of neutrinos with mass, but also the scale of energy at which the three fundamental forces of nature mentioned above are coupled together with equal strength. Below that energy, according to the standard model, the three forces can be differentially screened by virtual particles and antiparticles that flicker in and out of the vacuum. As Wilczek has pointed out, it must be more than coincidence that the unification energy inferred from neutrino masses, and that derived by theorists from the internal logic of the standard model, are the same, at around $10^{16} \mathrm{GeV}$. It may even point towards yet higher symmetries, the theories of which (supersymmetry, or SUSY) predict the existence of a plethora of particles that could well be observable using the next generation of particle accelerators.

All neutrinos seen so far are left-handed - their spin, if projected onto their direction of motion, is anticlockwise - whereas the spin of antineutrinos is right-handed. As Ed Witten explains (http://xxx.lanl.gov/abs/hep-ph/0006332), considerations of this 'helicity' and of neutrino mass can be related not only to a breakdown of a hypothetical conservation of quantum numbers (lepton and baryon numbers) ascribed to interacting particles, but also to quantum gravity theories that describe black holes and also the unification of gravity with the three other forces at energies of around $10^{19} \mathrm{GeV}$. Thus, according to theorists such as Witten and Wilczek, a knowledge of the nature of neutrino mixing brings us within theoretical spitting distance of the most elusive goal of all: the theory that unites all the fundamental forces of nature, or the Theory of Everything (TOE).

Particle experimentalists and the rest of us can at last welcome this beginning of an observational engagement with predictions of GUT, SUSY and TOE, via determinations, for example, of mass differences and oscillation probabilities, and inferences of the nature of the basic neutrino states. Whether generated in astronomical objects or in the atmosphere, or fired from near and distant accelerators, or from neutrino 'factories' that are still only gleams in experimentalists' eyes, mixed neutrinos provide us with a new lens through which to glimpse nature's deepest underlying structure. 